\title{
A Study on the Management Strategies of Classroom Teaching in Rural Junior Middle Schools under Role Perspective
}

\author{
Liu Junqiong
}

Huanggang Normal University, Huanggang city, Hubei Province, P.R.C. 438000,China

\begin{abstract}
Classroom teaching management is a complex and systematic activity process, involving teachers, students and the environment. Compared with cities, the "infrastructure" of rural schools has been improved, but the classroom management is confronting more difficulties. Consequently, students' comprehensive competences cannot be steadily improved. In order to improve the effectiveness and efficiency of the classroom teaching management, this article is based on the teaching ideology of "student-dominating, teacher-leading and trainingpenetrating", analyzing the different roles between students and teachers in the management, and proposes corresponding countermeasures so as to provide some useful references for effectively improving the teaching quality and students' all-round development.
\end{abstract}

Keywords:Classroom Teaching Management (CTM); Rural Junior Middle Schools (RJMSs); Classrooms of Rural Junior Middle School (CoRJMS)

With the comprehensive implementation of quality education in our country, it has been highly valued by governments at all levels and widely recognized by the people from all communities that the overall level of rural compulsory education should be effectively improved, and the relatively lagging situation of rural compulsory education must be changed. It is of great significance that the improvement of the educational situation in RJMSs has become an issue involving people's livelihood.

The National Medium and Long-term Educational Reform and Development Planning clearly

https://doi.org/10.47852/bonviewCETR2020010231

This is an open access article published by the BON VIEW PUBLISHING PTE. under the Creative Commons Attributions License. 
states that the schools should improve teachers' professional quality, teaching methods, classroom teaching effects and reduce the amount of homework and the number of exams, should cultivate students' learning interests and hobbies, strictly implement the curriculum plan, and must not increase class hours or increase difficulty. ${ }^{[1]}$ So it is necessary to improve the efficiency of classroom teaching to achieve those goals. Then the classroom teaching management becomes key.

What is CTM? It is very complicated. It is not only a dynamic process consisted of teachers and students who gather in the same physical space and time, but also a complex, changeable, vibrant and energetic system as a whole to achieve specific goals. Therefore, it refers to an activity process that teachers promote students to achieve the established goals through the interaction among teachers, students and the environment in the classroom. In CTM, teachers must ensure the order and efficiency, and coordinate and optimize the management objects to promote students' development, including cultivating students' autonomy and self-discipline, and paying attention to students' differences so that each student has space and opportunities to create and express himself. In this sense, the essence of classroom management is not only a result of achieving teaching goals, but also a process of using various strategies.

Compared with cities, although the "infrastructure" of RJMSs has been improved, the CTM confronting more difficulties. Consequently, the students' overall competence cannot be steadily improved. It involves many factors, but the primary factor is the teaching idea in the activities. The teaching ideology of "student-dominating, teacher-leading and training-penetrating" [2] should be applied in CTM. "Student-dominating" is the starting point in the activities and "teacher-leading" ensures the status of "student-dominating" is centered, since teacher and student is as a pair of basic categories. ${ }^{[3]}$ Moreover, "student-dominating" and "teacher-leading" are dialectically unified in the training penetrated in the teaching structure. This penetrating role of training plays to develop students' knowledge, skills, emotions, attitudes and values by teacher-student-environment interaction.

In reality, how does a teacher improve CTM? I hold that roles teachers and students play can be a good start as a clue, i.e. teacher's leading role and student's dominating role. Accordingly, I specifically propose the following coping strategies in two aspects:

\section{(1) Taking students as dominating roles}

Firstly, teachers must attract students with the dedicated spirit of professionalism. Lesson preparation, for example, it should be made "precisely, innovatively, practically and flexibly." All links must be deliberated and designed in preparation to be possibly best for the class. Assignments, another example, teachers should correct students' assignments promptly, especially focus on the underachievers' assignments to be fully checked and targeted to advise. It helps them not only duly make up for the knowledge lacked, but also gradually build confidence to cultivate their non-intellectual 
factors. With the preparation and assignment, thus, timely feedback and evaluation can be established to motivate teachers and students to reflect, leading the optimization of each link and all links in the teaching and learning, since the various links in the teaching process are interrelated and mutually cause and affect each other. The weakening or defect of any link will affect or even interrupt the normal operation of the teaching work. ${ }^{[4]}$

Teachers must attract students with morality and talents of professionalism. Due to students' relatively poor self-control, teachers' CTM has become very important. In the teaching, in order to attract and inspire students, teachers' talents play a key role in leading students into a wonderful world of knowledge. All those who are good at teaching pay attention to "racking their brains" before class, carefully preparing lessons so that they can master "outlines" (curriculum standards), "books" (textbooks), "people" (students), "knowing" and "methods" (strategies) in their minds. ${ }^{[5]}$ Only when teachers have strong talents in subject professionalism, can they thoroughly hold those five key elements, clearing the ideas thereby reflected and imparting what really "values". Thus, the students can be effectively taught in line with the actual needs of the teaching content and the practical conditions. That is, students' intelligence must be effectively promoted and their value judgment and realization in morality obtained with teachers' leading, especially under the enlightenment of the teachers' precepts and deeds.

Secondly, classroom activities must be participated in. The CTM purpose is not to control students, but to promote them to participate, moving and managing them with love. Classroom teaching activities are a process of interaction and cooperation between teachers and students, as well as a process of emotional communication. ${ }^{[6]}$ Human beings are emotional animals, and they can accumulate knowledge and experience in emotional interactions pleasantly. Chinese ancients said: "to be close to his teacher and believe in his way." In classroom, students' learning enthusiasm largely depends on the teachers. Teachers must care for students, paying attention to the close exchanges of recognition, affection, intention, and behavior, treating them with appreciation, and rewarding them with affirmation. In return, students are willing to be close to their teachers and participate in their activities. Notably, teachers must know about the internal needs of their students, and create a democratic, harmonious, relaxed, and free learning environment. It will not only increase the mutual intimacy, but also motivate students for more participation. In this way, the CTM can be better improved.

Thirdly, students' self-management must be guided by teachers. Suhomlinsky said: “True education is self-education that is the prerequisite and basis for self-management; self-management is the achievement and symbol of high-level self-education." In terms of it, we think that the highest state of CTM is to lead each student to carry out effective self-management. Students themselves can apply scientific management methods to self-regulate and self-control their own thoughts and behaviors under the guidance of the teachers in accordance with the education purposes and cultivation goals. ${ }^{[7]}$ Students 
should be led to continuously self-manage under teachers' targeted guidance. Only when students learn to self-manage can they possibly learn well.

\section{(2) Taking teachers as the leading roles}

Firstly, teachers' training should be adhered to. Teachers' training is the key to optimize CTM. Teachers should be encouraged to participate in different high-level academic training to get fostered. With the continuous improvement of professionalism via training, efficient teaching management is a matter of course.

Secondly, teachers' awareness and teaching views which are the soul of teaching should be changed. Guided by "students-dominating, teachers-leading and training-penetrating", it is not difficult to see that teachers are not only the designers of teaching activities, but also the instructors and participants. Students should be guided to dominate in the activities. Teaching purpose view of cultivating talents and task view of promoting students' morality, intelligence, physical fitness, aesthetics and labor must be established. Plus, teaching method view of taking heuristic teaching to inspire students in interaction must be also established. Commonly, the middle-class students and underachievers are basically the majority in RJMSs. It leaves people illusion that such students cannot be taught well. How teachers help them initiate and play the dominating role has become a more urgent and realistic topic. There are really differences in learning attitudes, methods, and habits compared with good students. But the practice has repeatedly proved that there are ways to teach them well. The key is to establish correct views. If teachers can take students as the center and mobilize their dominating roles, they can be greatly improved by training. The change of ideological concepts is fundamental, and the improvement of the level of ideology and theory will inevitably promote the improvement of teaching management. ${ }^{[8]}$

Teachers must have the awareness that teaching is also innovative and creative. Teachers' innovation and creation are not only the prerequisites for the implementation of curriculum standards, but also fundamental conditions for improving the teaching effectiveness. The internal logic of JMS knowledge is very rigorous with a wide range of points. Teachers should turn rigorous knowledge into vivid practice by every means, attracting students' interest and inspiring students to construct knowledge and form emotions, attitudes and values. Besides, innovating "two-role" model, and positioning the teacher-student relationship as companionship and transforming the traditional teacher's dominating role into student's dominating role to grasp the foundation of CTM. ${ }^{[9]}$ It manifests teachers' innovative awareness for effective practice. If teachers in RJMSs can establish this innovative awareness, the innovative teachers will occur. The teaching management of CoRJMS will naturally become efficient and effective. 
Thirdly, teaching research teachers should be cultivated. The overall level of CTM in RJMSs is low, and the low efficiency has led to the poor education quality and the imbalance of students' knowledge structure. Research teachers are required to change the situation and find the solutions. On the knowledge structure, teachers' research competence can be improved by openly integrating crossdisciplinary knowledge, broadening their vision and transforming thinking ways to improve classroom teaching. In this way, the value-added effect of classroom teaching in RJMSs will gradually emerge. The research teachers will increase.

Finally, scientific evaluation of CTM should be paid attention to. It is essential to build a multi-dimensional, comprehensive and dynamic evaluation system for quality improvement. The construction of the evaluation system should follow the principles of being scientific, practical, fairness, comprehensive, motivative and operable. ${ }^{[10]}$ It must firmly grasp and highlight the teaching quality, focus on classroom efficiency, and feedback on teaching results. It should not only satisfy the key indicators of compulsory education, but also truly achieve the overall development of students.

In short, CTM strategies are not isolated, and they are a system to each other. Only by overall planning and optimization of these strategies can the current situation of classroom teaching in RJMSs be efficiently changed, the goals of compulsory education of JMS be smoothly achieved, and the students' all-round development can go steadily.

\section{References}

[1] Ministry of Education. The National Medium and Long-term Educational Reform and Development Planning (2010-2020) [EB/OL]. http:// www.moe.edu.cn/ publicfiles/ business/ htmlfiles/ moe/moe_838/201008/93704.html.2010-07-29.

[2] Qian Menglong. Training--the Basic Form of Chinese Teaching. [J]. Chinese Teaching and Learning in Junior Middle School, 2010(01):24-27.

[3] Guo Yuanxiang. Educational Logic[M]. Beijing: People’s Education Press, 2019:228-228.

[4] [8] [10] Xu Wenrui. On the Teaching Management of Junior Middle Schools [J]. Education Reform,1995(05):32-33.

[5] [6] [7] Mo Lizhu. Classroom Management of Group Cooperative Learning in Geography Teaching in Junior Middle School [J]. Geography Teaching Reference for Middle School,2017(14):34-35.

[9] Xu Zhaolan. Research on the Management Strategy of Geography Classroom Teaching in Rural Junior Middle Schools[J]. Shanghai Educational Research, 2010(12):88-90. 